PROCEEDINGS OF THE

AMERICAN MATHEMATICAL SOCIETY

Volume 125, Number 3, March 1997, Pages 815-826

S 0002-9939(97)03631-9

\title{
ON NORMAL SOLVABILITY OF THE RIEMANN PROBLEM WITH SINGULAR COEFFICIENT
}

\author{
M. RAKOWSKI AND I. SPITKOVSKY \\ (Communicated by Palle E. T. Jorgensen)
}

\begin{abstract}
Suppose $G$ is a singular matrix function on a simple, closed, rectifiable contour $\Gamma$. We present a necessary and sufficient condition for normal solvability of the Riemann problem with coefficient $G$ in the case where $G$ admits a spectral (or generalized Wiener-Hopf) factorization $G_{+} \Lambda G_{-}$with $G_{-}^{ \pm 1}$ essentially bounded. The boundedness of $G_{-}^{ \pm 1}$ is not required when $G$ takes injective values a.e. on $\Gamma$.
\end{abstract}

\section{INTRODUCTION}

Let $\Gamma$ be a rectifiable, positively oriented boundary of a finitely connected domain $\mathcal{D}_{+} \subset \mathbf{C}$, and let $\mathcal{D}_{-}=\mathbf{C}_{\infty} \backslash\left(\mathcal{D}_{+} \cup \Gamma\right)$. If $1 \leq p \leq \infty$, let $E_{p+}$ and $E_{p-}$ be the usual Smirnov spaces associated with the domains $\mathcal{D}_{+}$and $\mathcal{D}_{-}$(see e.g. [5]). That is, $E_{\infty+}$ is the space of functions analytic and bounded in $\mathcal{D}_{+}$, and, if $1 \leq p<\infty, E_{p+}$ contains functions $f$ analytic in $\mathcal{D}_{+}$for which there exists an expanding sequence of regions $\mathcal{D}_{k}$ with rectifiable boundaries $\Gamma_{k}$ such that

(i) $\mathcal{D}_{k} \cup \Gamma_{k} \subset \mathcal{D}_{+}$,

(ii) $\bigcup \mathcal{D}_{k}=\mathcal{D}_{+}$,

(iii) $\sup \int_{\Gamma_{k}}|f(t)|^{p} d t<\infty$.

The spaces $E_{p-}(1 \leq p \leq \infty)$ are defined by replacing $\mathcal{D}_{+}$with $\mathcal{D}_{-}$. We will denote by $\dot{E}_{p-}$ the subspace of $E_{p-}$ formed by functions vanishing at infinity.

A function $f \in E_{p+}$ (or $E_{p-}$ ) has a nontangential boundary limit at a.e. $t \in \Gamma$. These limits define a function $\tilde{f} \in L_{p}(\Gamma)=: L_{p}$. The map $f \rightarrow \tilde{f}$ is injective, and we will identify $E_{p \pm}$ with $L_{p \pm}$, the space of boundary values of functions in $E_{p \pm}$. We will write $\dot{L}_{p}$ instead of $\dot{E}_{p}$. $\mathcal{R}$ will denote the space of rational functions without poles on $\Gamma$. If $X \in\left\{L_{p+}, L_{p-}, \dot{L}_{p-}, \mathcal{R}\right\}$, we will denote the space of $m \times n$ matrices over $X$ by $X^{m \times n}$. If $m=1$ or $n=1$, we will write $X^{n}$ or $X^{m}$.

Let $p \in(1, \infty)$, let $g \in L_{p}^{m}$, and suppose $G$ is an $m \times n$ matrix valued function on $\Gamma$ with measurable entries. The Riemann problem consists in finding functions $\phi_{+} \in L_{p+}^{m}$ and $\phi_{-} \in \dot{L}_{p-}^{n}$ such that

$$
\phi_{+}+G \phi_{-}=g .
$$

Received by the editors September 8, 1995.

1991 Mathematics Subject Classification. Primary 45E05, 45F15, 47A68.

This research was partially supported by the NSF Grants DMS-9302706 and DMS-9401848.

(c)1997 American Mathematical Society 
If $q=p /(p-1)$ and $h \in L_{q}^{n}$, the dual problem consists in finding $\psi_{+} \in L_{q+}^{m}$ and $\psi_{-} \in \dot{L}_{q-}^{n}$ such that

$$
\psi_{-}+G^{T} \psi_{+}=h .
$$

The image of the Riemann problem (or the dual problem), i.e. the set of all $g \in L_{p}^{m}$ (or $h \in L_{q}^{n}$ ) for which the problem is consistent, is a linear manifold. We present a condition for the problem to be normally solvable, i.e., for the image of the problem to be closed.

The Riemann problem with nonsingular coefficient has been extensively studied in the literature-see [2] or [6] and the references cited there. One can consider a more general problem where the coefficient takes singular values. Suppose a zero row or column has been appended to a nonsingular matrix valued function. Even in this simple case the theory developed for nonsingular coefficients cannot be applied directly, although the problem may be well posed and have a unique solution for a given $g$. Plainly, one can have more general coefficients $G$.

The Riemann problem can be reformulated in terms of singular integral equations. Let $\mathcal{S}: L_{p} \rightarrow L_{p}$ be the operator of singular integration along $\Gamma$, i.e.,

$$
\left.(\mathcal{S} f)(t)=\frac{1}{\pi i} \int_{\Gamma} \frac{f(\tau)}{\tau-t} d \tau\right)
$$

with the integral understood in the sense of the principal value. Suppose the contour $\Gamma$ is such that $\mathcal{S}$ is continuous on the whole space $L_{p}$. Then $\mathcal{P}=(I+\mathcal{S}) / 2$ and $\mathcal{Q}=I-\mathcal{P}$ are continuous complementary projections with the images $L_{p+}$ and $\dot{L}_{p-}$, respectively, and (1.1) can be written as $\mathcal{P} \pi_{m} \phi+G \mathcal{Q} \pi_{n} \phi=g$ where $\mathcal{P}$ and $\mathcal{Q}$ act componentwise and $\pi_{j}$ selects the first $j$ components of $\phi, j=m, n$. That is, (1.1) is equivalent to the system of singular integral equations with Cauchy kernel

$$
\left\{\begin{array}{c}
\phi_{1}(t)+\frac{1}{\pi i} \int_{\Gamma} \frac{\phi_{1}(\tau)}{\tau-t} d \tau+G_{11}(t)\left(\phi_{1}(t)-\frac{1}{\pi i} \int_{\Gamma} \frac{\phi_{1}(\tau)}{\tau-t} d \tau\right) \\
+\ldots+G_{1 n}(t)\left(\phi_{n}(t)-\frac{1}{\pi i} \int_{\Gamma} \frac{\phi_{n}(\tau)}{\tau-t} d \tau\right)=2 g_{1}(t) \\
\phi_{2}(t)+\frac{1}{\pi i} \int_{\Gamma} \frac{\phi_{2}(\tau)}{\tau-t} d \tau+G_{21}(t)\left(\phi_{1}(t)-\frac{1}{\pi i} \int_{\Gamma} \frac{\phi_{1}(\tau)}{\tau-t} d \tau\right) \\
+\ldots+G_{2 n}(t)\left(\phi_{n}(t)-\frac{1}{\pi i} \int_{\Gamma} \frac{\phi_{n}(\tau)}{\tau-t} d \tau\right)=2 g_{2}(t) \\
\vdots \quad \\
\phi_{m}(t)+\frac{1}{\pi i} \int_{\Gamma} \frac{\phi_{m}(\tau)}{\tau-t} d \tau+G_{m 1}(t)\left(\phi_{1}(t)-\frac{1}{\pi i} \int_{\Gamma} \frac{\phi_{1}(\tau)}{\tau-t} d \tau\right) \\
+\ldots+G_{m n}(t)\left(\phi_{n}(t)-\frac{1}{\pi i} \int_{\Gamma} \frac{\phi_{n}(\tau)}{\tau-t} d \tau\right)=2 g_{m}(t)
\end{array}\right.
$$

Here, the functions $G_{i j}$ and $g_{i}$ are given, and one looks for a solution $\phi_{1}, \phi_{2}, \ldots$, $\phi_{\max (m, n)} \in L_{p}$. In this system, the coefficient $G=\left[G_{i j}\right]$ may be rectangular or square with the determinant zero a.e. on $\Gamma$. We are interested in the question when the set of $m$-tuples $\left(g_{1}, g_{2}, \ldots, g_{m}\right)$ for which the system is consistent forms a closed subspace of $L_{p}^{m}$.

We end the introduction with a remark on contours. If $1 \leq p \leq \infty, L_{p+}$ and $L_{p-}$ are closed subspaces of $L_{p}$ whose sum is dense in $L_{p}$. Also, $L_{p+} \cap \dot{L}_{p-}=(0)$. 
Suppose $1<p<\infty$. Then $L_{p}=L_{p+} \dot{+} \dot{L}_{p-}$ if and only if $\Gamma$ is regular [4], i.e., if and only if the quotient of the length of $\Gamma$ inside any circle to the radius of the circle is less than some fixed constant. If this condition is satisfied, the projections $\mathcal{P}$ and $\mathcal{Q}$ are continuous. A regular contour is Smirnov, i.e., the derivative of the conformal mapping which maps the unit disk onto $\mathcal{D}_{+}$if $\mathcal{D}_{+}$is simply connected, or onto any connected component of $\mathcal{D}_{-}$, belongs to the Hardy space $H_{1}$. Below, we will not assume that $\Gamma$ is regular or Smirnov.

\section{Spectral Factorization}

Let $G$ be an $m \times n$ matrix function on $\Gamma$ with measurable entries. By a (left) spectral factorization in $L_{p}$ we will understand a factorization

$$
G=G_{+} \Lambda G_{-}
$$

where

(i) $G_{+} \in L_{p+}^{m \times m}$ has nonsingular values a.e. on $\Gamma$ and $G_{+}^{-1} \in L_{q+}^{m \times m}$,

(ii) $G_{-} \in L_{q-}^{n \times n}$ has nonsingular values a.e. on $\Gamma$ and $G_{-}^{-1} \in L_{p-}^{n \times n}$,

(iii) the middle factor

$$
\Lambda(t)=\left[\begin{array}{cc}
\operatorname{diag}\left(\left(\frac{t-t_{+}}{t-t_{-}}\right)^{\kappa_{1}}, \ldots,\left(\frac{t-t_{+}}{t-t_{-}}\right)^{\kappa_{k}}\right) & 0 \\
0 & 0
\end{array}\right]
$$

for some $t_{+} \in \mathcal{D}_{+}$and $t_{-} \in \mathcal{D}_{-}$and integers $\kappa_{1} \geq \kappa_{2} \geq \ldots \geq \kappa_{k}$.

Note that we do not require the operator $G_{+} \mathcal{P} G_{+}^{-1}$ to be bounded (cf. [2]; see also [1]). Also note that if the factorization (2.1) exists, $k=\operatorname{rank} G(t)$ for a.e. $t \in \Gamma$. In particular, $k$ is unique.

To simplify notation, we will assume $0 \in \mathcal{D}_{+}$and replace $\left(\frac{t-t_{+}}{t-t_{-}}\right)$by $t$, a factor which vanishes at the origin and has a pole at infinity. Then (2.2) becomes

$$
\Lambda(t)=\left[\begin{array}{cc}
\operatorname{diag}\left(t^{\kappa_{1}}, t^{\kappa_{2}}, \ldots, t^{\kappa_{k}}\right) & 0 \\
0 & 0
\end{array}\right]
$$

an $m \times n$ matrix function with $k$ nonzero entries.

Our definition of (left) spectral factorization differs from that in $[3,7]$ and [8] where it is required that $G_{+} \in L_{p+}^{m \times k}$ have a multiplicative left inverse in $L_{q+}^{k \times m}, G_{-} \in L_{q-}^{k \times n}$ have a multiplicative right inverse in $L_{p-}^{n \times k}$, and that $\Lambda(t)=$ $\operatorname{diag}\left(t^{\kappa_{1}}, t^{\kappa_{2}}, \ldots, t^{\kappa_{k}}\right)$. In fact, our definition is stronger. If $G$ has a spectral factorization in $L_{p}$ in the sense above, the spectral factorization as in $[3,7]$ and [8] can be obtained by deleting columns of $G_{+}$and rows of $G_{-}$corresponding to the zero rows or columns of $\Lambda$, and replacing $\Lambda$ by its nonsingular upper left block.

In particular, Theorem 2.2 in [8] has the following analogue for spectral factorization defined above.

Theorem 2.1. Suppose $1<p_{1} \leq p_{2}<\infty$ and let $G_{1+} \Lambda_{1} G_{1-}$ and $G_{2+} \Lambda_{2} G_{2-}$ with

$$
\Lambda_{j}(t)=\left[\begin{array}{cc}
\operatorname{diag}\left(t^{\kappa_{1}^{(j)}}, t_{2}^{\kappa_{2}^{(j)}}, \ldots, t^{\kappa_{k}^{(j)}}\right) & 0 \\
0 & 0
\end{array}\right], \quad j=1,2,
$$

be spectral factorizations in $L_{p_{1}}$ and $L_{p_{2}}$ of a function $G$ relative to a contour $\Gamma$. Then $\kappa_{j}^{(1)} \geq \kappa_{j}^{(2)}$ for $j=1,2, \ldots, k$. 
It follows from Theorem 2.1 that the integers $\kappa_{i}$ in (2.3) are unique. They are called the indices of the factorization. The sum $\sum_{i=1}^{k} \kappa_{i}$ is the total index of the factorization. If all indices of the factorization are equal to 0 , the factorization is said to be canonical.

Nonuniqueness of the factorization can be characterized as follows.

Theorem 2.2. Suppose $G=G_{+} \Lambda G_{-}$with

$$
\Lambda(t)=\left[\begin{array}{cc}
\operatorname{diag}\left(t^{\kappa_{1}}, t^{\kappa_{2}}, \ldots, t^{\kappa_{k}}\right) & 0 \\
0 & 0
\end{array}\right]=:\left[\begin{array}{cc}
\hat{\Lambda}(t) & 0 \\
0 & 0
\end{array}\right]
$$

is a spectral factorization in $L_{p}$ of a function $G$ relative to $\Gamma$. Then $G_{2+} \Lambda G_{2-}$ is a spectral factorization of $G$ in $L_{p}$ relative to $\Gamma$ if and only if

$$
G_{2+}=G_{1+}\left[\begin{array}{cc}
H_{+} & A_{+} \\
0 & B_{+}
\end{array}\right] \quad \text { and } \quad G_{2-}=\left[\begin{array}{cc}
\hat{\Lambda}^{-1} H_{+}^{-1} \hat{\Lambda} & 0 \\
A_{-} & B_{-}
\end{array}\right] G_{1-}
$$

where $H_{+}$of size $k \times k$ is a unimodular matrix polynomial such that

(i) $h_{i j}=0$ if $\kappa_{i}<\kappa_{j}$,

(ii) $\operatorname{deg} h_{i j} \leq \kappa_{i}-\kappa_{j}$ if $\kappa_{i} \geq \kappa_{j}$,

and $A_{+}, B_{+}$with entries in $L_{1+}$, and $A_{-}, B_{-}$with entries in $L_{1-}$, are such that $G_{2+} \in L_{p+}^{m \times m}, G_{2+}^{-1} \in L_{q+}^{m \times m}, G_{2-} \in L_{q-}^{n \times n}, G_{2-}^{-1} \in L_{p-}^{n \times n}$.

The properties of $H_{+}$follow from Theorem 2.4 in [8]. Since $G_{2+}=G_{1+}\left(G_{1+}^{-1} G_{2+}\right)$ and $G_{2-}=\left(G_{2-} G_{1-}^{-1}\right) G_{1-}, A_{ \pm}$and $B_{ \pm}$have entries in $L_{1 \pm}$. Note that, unlike with the definition of spectral factorization in [8], $G$ may admit a spectral factorization in $L_{p_{1}}$ and $L_{p_{2}}\left(p_{1}<p_{2}\right)$ with the same total index, but the factorization in $L_{p_{1}}$ may fail to be a factorization in $L_{p_{2}}$. A simple example is provided by

$$
G(t)=\left[\begin{array}{cc}
1 & 0 \\
0 & \phi(t)
\end{array}\right]\left[\begin{array}{l}
t \\
0
\end{array}\right][1]
$$

where $\phi \in L_{p_{1}+} \backslash L_{p_{2}+}$ and $\phi^{-1} \in L_{q_{1}+}$.

\section{RiEMANN PROBLEM WITH A FACTORABLE COEFFICIENT}

We will assume in this section that the coefficient $G$ of the Riemann problem (1.1) admits spectral factorization in $L_{p}$ relative to $\Gamma$ with the middle factor $\Lambda$ as in (2.3). $\Lambda^{\dagger}$ will denote the pointwise Moore-Penrose inverse of $\Lambda$,

$$
\Lambda^{\dagger}(t)=\left[\begin{array}{cc}
\operatorname{diag}\left(t^{-\kappa_{1}}, t^{-\kappa_{2}}, \ldots, t^{-\kappa_{k}}\right) & 0 \\
0 & 0
\end{array}\right] .
$$

If $G_{-} \Lambda G_{+}$is a spectral factorization in $L_{p}$ of a nonsingular matrix function $G$, $g$ belongs to the image of the Riemann problem with coefficient $G$ if and only if

$$
G_{+}^{-1} g \in L_{1+}^{n} \dot{+} L_{1-}^{n}, \quad G_{+} \mathcal{P} G_{+}^{-1} g \in L_{p+}^{n}, \quad G_{-}^{-1} \Lambda^{-1} \mathcal{Q} G_{+}^{-1} g \in \dot{L}_{p-}^{n} .
$$

If $G$ takes singular values, the last relation does not have to be valid. Indeed, suppose $\Gamma$ is the unit circle, $p=\frac{3}{2}, q=3$, and

$$
G(t)=I\left[\begin{array}{ll}
1 & 0 \\
0 & 0
\end{array}\right]\left[\begin{array}{cc}
\left(\frac{t-1}{t}\right)^{\frac{1}{4}} & 0 \\
1 & \left(\frac{t-1}{t}\right)^{\frac{1}{4}}
\end{array}\right]=: G_{+}(t) \Lambda(t) G_{-}(t)
$$


for some branch of $\left(\frac{z-1}{z}\right)^{\frac{1}{4}}$ on $\mathbf{C}_{\infty} \backslash[0,1]$, so that $G_{-} \in L_{3-}^{2 \times 2}$ and $G_{-}^{-1} \in L_{\frac{3}{2}-}^{2 \times 2}$. The function

$$
g(t)=\left[\begin{array}{c}
\frac{1}{t}\left(\frac{t}{t-1}\right)^{\frac{1}{4}} \\
0
\end{array}\right]
$$

belongs to the image of the problem, but

$$
\left(G_{-}^{-1} \Lambda^{\dagger} \mathcal{Q} G_{+}^{-1} g\right)(t)=\left[\begin{array}{c}
\frac{1}{t}\left(\frac{t}{t-1}\right)^{\frac{1}{2}} \\
-\frac{1}{t}\left(\frac{t}{t-1}\right)^{\frac{3}{4}}
\end{array}\right] \notin L_{\frac{3}{2}}^{2 \times 1} .
$$

Proposition 3.1. Suppose $G=G_{+} \Lambda G_{-}$with $\Lambda \in L_{\infty}^{m \times n}$ as in (2.3) is a spectral factorization in $L_{p}$ and let $g \in L_{p}^{m}$. Then the problem $\phi_{+}+G \phi_{-}=g$ is solvable if and only if

$$
G_{+}^{-1} g \in L_{1+}^{m} \dot{+}\left[\begin{array}{c}
\dot{L}_{1-}^{k} \\
0
\end{array}\right], \quad G_{+} \mathcal{P} G_{+}^{-1} g \in L_{p+}^{m}
$$

and

$$
G_{-}^{-1}\left(\Lambda^{\dagger} \mathcal{Q} G_{+}^{-1} g+r\right) \in \dot{L}_{p-}^{n}
$$

for some $r \in \dot{L}_{1-}^{n}$ with the first $k$ components zero. If the problem is solvable, then the general solution has the form

$$
\left(G_{+}\left(\mathcal{P} G_{+}^{-1} g+\rho\right), G_{-}^{-1}\left(\Lambda^{\dagger} \mathcal{Q} G_{+}^{-1} g-\Lambda^{\dagger} \rho+r\right)\right)
$$

where $\rho$ is a vector function whose $j^{\text {th }}$ entry is zero if $j>k$ or $\kappa_{j} \leq 0$, and a polynomial of degree at most $\kappa_{j}-1$ otherwise, and $r \in \dot{L}_{1-}^{n}$ is a function with the first $k$ components zero and such that (3.2) holds. Moreover, if $G_{-}^{ \pm 1} \in L_{\infty}^{n \times n},(3.2)$ and (3.3) can be replaced by

$$
G_{-}^{-1} \Lambda^{\dagger} \mathcal{Q} G_{+}^{-1} g \in \dot{L}_{p-}^{n}
$$

and

$$
\left(G_{+}\left(\mathcal{P} G_{+}^{-1} g+\rho\right), G_{-}^{-1}\left(\Lambda^{\dagger} \mathcal{Q} G_{+}^{-1} g-\Lambda^{\dagger} \rho\right)\right) .
$$

Proof. Suppose $\left(\phi_{+}, \phi_{-}\right)$solves the problem. Since $f_{+}:=G_{+}^{-1} \phi_{+} \in L_{1+}^{m}$ and $f_{-}:=G_{-} \phi_{-} \in \dot{L}_{1-}^{n}, G_{+}^{-1} g \in L_{1+}^{m} \dot{+} \dot{L}_{1-}^{m}$. Since $\Lambda f_{-}$has zero entries in the bottom $m-k$ components, the first relation in (3.1) is valid. Also,

$$
\rho:=f_{+}-\mathcal{P} G_{+}^{-1} g=-\Lambda f_{-}+\mathcal{Q} G_{+}^{-1} g
$$

is a vector polynomial satisfying the degree requirements. Since $\phi_{+}=G_{+} \mathcal{P} G_{+}^{-1} g+$ $G_{+} \rho$, the second relation in (3.1) holds. Since

$$
\Lambda G_{-} \phi_{-}=\mathcal{Q} G_{+}^{-1} g-\rho,
$$

$\Lambda^{\dagger} \rho \in \dot{L}_{\infty-}^{n}$, and the bottom $n-k$ components of $G_{-} \phi_{-}$are in $L_{1},(3.2)$ holds (with $r$ agreeing with $G_{-} \phi_{-}$in the bottom $n-k$ positions).

Conversely, suppose (3.1) and (3.2) (or (3.2')) hold. If

$$
\left(\varphi_{+}, \varphi_{-}\right)=\left(G_{+} \mathcal{P} G_{+}^{-1} g, G_{-}^{-1} \Lambda^{\dagger} \mathcal{Q} G_{+}^{-1} g+G_{-}^{-1} r\right)
$$

for an appropriate $r$,

$$
\varphi_{+}+G \varphi_{-}=G_{+} \mathcal{P} G_{+}^{-1} g+G_{+} \Lambda \Lambda^{\dagger} \mathcal{Q} G_{+}^{-1} g=G_{+} \mathcal{P} G_{+}^{-1} g+G_{+} \mathcal{Q} G_{+}^{-1} g=g
$$


Since $\left(G_{+} \rho,-G_{-}^{-1} \Lambda^{\dagger} \rho\right)$ with $\rho$ a vector polynomial satisfying the degree requirements is a solution of the homogeneous problem, (3.3) solves the problem. Let $\phi_{-} \in \dot{L}_{p-}^{n}$ be such that $G \phi_{-} \in L_{p+}^{m}$. Then $\Lambda G_{-} \phi_{-} \in L_{1+}^{m}$, and $\phi_{-}=G_{-}^{-1}\left(\Lambda^{\dagger} \rho+r\right)$ with $\rho$ and $r$ as in the statement of the theorem. Since $G\left(G_{-}^{-1}\left(\Lambda^{\dagger} \rho+r\right)\right)=G_{+} \rho$, it follows that (3.3) is the general solution of the problem.

Suppose $G_{-}^{ \pm 1} \in L_{\infty}^{n \times n}$ and $\left(\phi_{+}, \phi_{-}\right)$is a solution of the problem. Then

$$
\Lambda^{\dagger} \Lambda G_{-} \phi_{-}=\Lambda^{\dagger} \Lambda G_{-}\left(G_{-}^{-1} G_{-} \phi_{-}\right)=\Lambda^{\dagger} \Lambda G_{-} G_{-}^{-1} \tilde{\phi}_{-}
$$

where $\tilde{\phi}_{-} \in \dot{L}_{p-}^{n}$ has zero $n-k$ bottom components. Hence (3.4) implies

$$
G_{-}\left(G_{-}^{-1} \tilde{\phi}_{-}\right)=\Lambda^{\dagger} \mathcal{Q} G_{+}^{-1} g-\Lambda^{\dagger} \rho
$$

and $\left(3.2^{\prime}\right)$ holds.

Note that if $G$ takes injective values a.e. on $\Gamma, k=n$ and $\left(3.2^{\prime}\right)$ and $\left(3.3^{\prime}\right)$ can be used instead of (3.2) and (3.3). Also note that in the example before Proposition 3.1 , the restriction of the operator $\Lambda \Lambda^{\dagger} G_{-}^{-1} \Lambda^{\dagger} \mathcal{Q} G_{+}^{-1}$ to the image of the problem was bounded in $L_{p}^{2}$. However, this need not be true in the general case. Indeed, suppose $p, q$, and $\Gamma$ are as in the example preceding Proposition 3.1 and

$$
G(t)=I\left[\begin{array}{ll}
1 & 0 \\
0 & 0
\end{array}\right]\left[\begin{array}{cc}
0 & 1 \\
\left(\frac{t-1}{t}\right)^{\frac{1}{4}} & \left(\frac{t-1}{t}\right)^{-\frac{1}{4}}
\end{array}\right]=: G_{+}(t) \Lambda(t) G_{-}(t)
$$

for some branch of $\left(\frac{z-1}{z}\right)^{\frac{1}{4}}$ on $\mathbf{C}_{\infty} \backslash[0,1]$. Then

$$
g(t)=\left[\begin{array}{c}
\frac{1}{t}\left(\frac{t}{t-1}\right)^{\frac{1}{4}} \\
0
\end{array}\right]=G\left[\begin{array}{c}
0 \\
\frac{1}{t}\left(\frac{t}{t-1}\right)^{\frac{1}{4}}
\end{array}\right]
$$

belongs to the image of the problem, but

$$
\left(\Lambda \Lambda^{\dagger} G_{-}^{-1} \Lambda^{\dagger} \mathcal{Q} G_{+}^{-1} g\right)(t)=\left[\begin{array}{c}
-\frac{1}{t}\left(\frac{t}{t-1}\right)^{\frac{3}{4}} \\
0
\end{array}\right] \notin L_{\frac{3}{2}}^{2 \times 1} .
$$

To simplify notation, we will write $X^{-T}$ and $X^{\dagger T}$ instead of $\left(X^{-1}\right)^{T}$ and $\left(X^{\dagger}\right)^{T}$ for any matrix (or matrix valued function) $X$. Rewriting the dual equation as $G_{-}^{-T} \psi_{-}+\Lambda^{T} G_{+}^{T} \psi_{+}=G_{-}^{-T} h$, one can show the following dual version of Proposition 3.1 .

Proposition 3.2. Suppose $G=G_{+} \Lambda G_{-}$with $\Lambda$ as in (2.3) is a spectral factorization in $L_{p}$. Then $h \in L_{q-}^{n}$ belongs to the image of the dual problem if and only if

$$
G_{-}^{-T} h \in\left[\begin{array}{c}
L_{1+}^{k} \\
0
\end{array}\right] \dot{+} \dot{L}_{1-}^{n}, \quad G_{-}^{T} Q G_{-}^{-T} h \in \dot{L}_{q-}^{n}, \quad G_{+}^{-T}\left(\Lambda^{\dagger T} \mathcal{P} G_{-}^{-T} h+r\right) \in L_{q+}^{n}
$$

for some $r \in L_{1+}^{m}$ with the first $k$ components zero. If the conditions (3.5) are satisfied, then the general solution of the dual problem has the form

$$
\left(G_{+}^{-T}\left(\Lambda^{\dagger T} \mathcal{P} G_{-}^{-T} h-\Lambda^{\dagger T} \rho+r\right), G_{-}^{T}\left(\mathcal{Q} G_{-}^{-T} h+\rho\right)\right)
$$

where $\rho$ is a vector function with $j^{\text {th }}$ entry zero if $j>k$ or $\kappa_{j} \geq 0$, and a polynomial in $z^{-1}$ of order at most $-\kappa_{j}$ without a constant term otherwise, and $r \in L_{1+}^{m \times 1}$ with zeros in the first $k$ positions is such that the last relation in (3.5) holds. Moreover, if $G_{+}^{ \pm 1} \in L_{\infty}^{m \times m}$, the statement holds with $r=0$. 
If $1 \leq p<\infty$, we will identify $L_{q}^{n}$ with the dual space of $L_{p}^{n}$ through the scalar product

$$
\langle f, g\rangle=\sum_{j=1}^{n} \int_{\Gamma} f_{j}(t) g_{j}(t) d t
$$

for all $f(t)=\sum_{j=1}^{n} f_{j}(t) e_{j} \in L_{p}^{n}$ and $g(t)=\sum_{j=1}^{n} g_{j}(t) e_{j} \in L_{q}^{n}$.

Theorem 3.3. Suppose $G=G_{+} \Lambda G_{-}$is a spectral factorization in $L_{p}$ with $\Lambda$ as in (2.3). Then

(i) $g \in L_{p}^{m}$ belongs to the closure of the image of the problem if and only if

$$
\int_{\Gamma} e_{j}^{T} G_{+}^{-1}(t) g(t) t^{i} d t=0 \quad \text { for } j \leq k \text { such that } \kappa_{j}<0 \text { and } i=0,1, \ldots,-\kappa_{j}-1
$$

$$
e_{j}^{T} G_{+}^{-1}(t) g(t) \in L_{1+} \quad(j=k+1, k+2, \ldots, m),
$$

(ii) $h \in L_{q}^{n}$ belongs to the closure of the image of the dual problem if and only if

$$
\begin{aligned}
& \int_{\Gamma} e_{j}^{T} G_{-}^{-T}(t) h(t) t^{i} d t=0 \quad \text { for } j \leq k \text { such that } \kappa_{j}>0 \text { and } i=-1,-2, \ldots,-\kappa_{j} \\
& \quad \text { and } \\
& \quad e_{j}^{T} G_{-}^{-T}(t) h(t) \in \dot{L}_{1-} \quad(j=k+1, k+2, \ldots, n) .
\end{aligned}
$$

Proof. We verify (i). The annihilator of the image of the problem contains "+" components of elements in the kernel of the dual problem. Since, if $\kappa_{j}<0$,

$$
\left(z^{i} G_{+}^{-T}(z) e_{j},-z^{i} G_{-}^{T}(z) \Lambda^{T}(z) e_{j}\right) \quad\left(i=0,1, \ldots,-\kappa_{j}-1\right)
$$

is in the kernel of the dual problem, (3.6) holds for each $g$ in the image of the problem $\left(i=0,1, \ldots,-\kappa_{j}-1\right)$. If $j>k$, where $k$ is the rank of $G$ a.e. on $\Gamma$, $\left(G_{+}^{-1}(z) z^{i} e_{j}, 0\right)$ belongs to the kernel of the dual problem for $i \geq 0$. Hence, for each $g$ in the image of the problem,

$$
\int_{\Gamma} e_{j}^{T} G_{+}^{-1}(t) g(t) t^{i} d t=0 \quad(i=0,1,2, \ldots)
$$

and (3.7) holds. Thus, the space of $g \in L_{p}^{m}$ which satisfy (3.6) and (3.7) contains the closure of the image of the problem.

To show the converse, we need to identify the dual of the space

$$
\left\{g \in L_{p}^{m}: e_{j}^{T} G_{+}^{-1}(t) g(t) \in L_{1+} \text { for } j=k+1, k+2, \ldots, m\right\} .
$$

First note that $\mathcal{R}^{1 \times m} G_{+}^{-1}$ (where $\mathcal{R}$ denotes the space of rational functions analytic on $\Gamma$ ) is dense in $L_{q}^{m}$. Indeed, if $\phi \in L_{p}^{m}$ is such that $\left\langle r G_{+}^{-1}, \phi\right\rangle=0$ for all $r \in \mathcal{R}^{1 \times m}$, $\left\langle r, G_{+}^{-1} \phi\right\rangle=0$ for all $r \in \mathcal{R}^{1 \times m}$ and so $G_{+}^{-1} \phi=0$ a.e. on $\Gamma$. Thus, $\phi=0$. Now

$$
\left[0 \quad \mathcal{R} L_{\infty+}^{1 \times(m-k)}\right] G_{+}^{-1}
$$

(where $\mathcal{R} L_{\infty \pm}^{n}=\mathcal{R}^{n} \cap L_{\infty \pm}^{n}$ ) annihilates (3.10). If

$$
0 \neq \psi \in\left[\begin{array}{ll}
\mathcal{R}^{1 \times k} & \mathcal{R} L_{\infty-}^{1 \times(m-k)}
\end{array}\right] G_{+}^{-1},
$$


we can find $r \in \mathcal{R}^{m}$ such that $G_{+} r$ belongs to (3.10) but $\left\langle\psi, G_{+} r\right\rangle \neq 0$. Thus, the annihilator of the space (3.10) coincides with the $L_{q}^{m}$-closure of (3.11) and

$$
L_{q}^{m} / \operatorname{cl}\left(\left[\begin{array}{ll}
0 & \mathcal{R} L_{\infty+}^{1 \times(m-k)}
\end{array}\right] G_{+}^{-1}\right)
$$

is the dual of (3.10).

It is now easy to show that

$$
G_{+}\left[\begin{array}{c}
\mathcal{R}^{k \times 1} \\
\mathcal{R} L_{\infty+}^{(m-k) \times 1}
\end{array}\right]
$$

is dense in (3.10). Indeed, suppose an element $\phi$ of (3.13) annihilates (3.14), and let $\psi_{k}$ be a sequence of elements as in (3.12) which converges to $\phi$. Then each $\psi_{k}$ annihilates (3.14), and so $\psi_{k}=0$ for all $k$. Thus, $\phi=0$.

The elements of (3.14) satisfy conditions (3.1). If $g \in L_{p}^{m}$ satisfies (3.6), then $e_{j}^{T} \mathcal{Q} G_{+}^{-1} g$ must have a zero at infinity of order at least $\kappa_{j}+1$ (cf. Lemma 3.1 in [6]). Thus, the members of (3.14) for which (3.6) holds satisfy also (3.2') and hence belong to the image of the problem. The subspace of (3.10) formed by elements which satisfy (3.6) has a finite co-dimension, equal to the sum of positive indices of the factorization, and one can choose a basis for its complement in the set (3.14). It follows that the image of the problem contains a subset dense in the space of functions $g \in L_{p}^{m}$ which satisfy (3.6) and (3.7).

In view of the last theorem, Propositions 3.1 and 3.2 have the following corollary.

Corollary 3.4. If $G$ admits a spectral factorization in $L_{p}$, then the image of the Riemann problem (or the dual problem) contains all rational vector functions in its closure as a dense subset.

Proof. Let $g$ be a rational $m \times 1$ vector function. Then

$$
G_{+}^{-1} g \in L_{1+}^{m} \dot{+} \dot{L}_{1-}^{m}, \quad G_{+} \mathcal{P} G_{+}^{-1} g \in L_{p+}^{m}, \quad \mathcal{Q} G_{+}^{-1} g \in \dot{L}_{\infty-}^{m} .
$$

If $g$ belongs to the closure of the image of the problem, then (3.7) and (3.15) imply (3.1). By (3.6), $e_{j}^{T} \mathcal{Q} G_{+}^{-1} g$ has a zero at infinity of order $-\kappa_{j}+1$, so that $\left(3.2^{\prime}\right)$ is also valid and $g$ belongs to the image of the problem. To verify density, note that the elements $\phi$ of (3.14) which satisfy (3.6) are dense in the image of the problem. For any such $\phi, \mathcal{Q} \phi$ is rational and $\mathcal{P} \phi$ belongs to the $L_{p}^{m}$ closure of $\mathcal{R} L_{\infty+}^{m}$.

The proof for the dual problem is analogous.

\section{Normal solvability}

The kernel of the Riemann problem with coefficient $G$ is isomorphic to

$$
\begin{aligned}
& \left\{\left(\phi_{+}, \phi_{-}\right) \in L_{p+}^{m} \times \dot{L}_{p-}^{n}: \phi_{+}+G \phi_{-}=0\right\} \cong\left\{\phi_{-} \in \dot{L}_{p-}^{n}: G \phi_{-} \in L_{p+}^{m}\right\} \\
& \quad \cong\left\{\phi_{+} \in L_{p+}^{m}: \phi_{+}+G \phi_{-}=0 \text { for some } \phi_{-} \in \dot{L}_{p-}^{n}\right\} \dot{+}\left\{\phi_{-} \in \dot{L}_{p-}^{n}: G \phi_{-}=0\right\} .
\end{aligned}
$$

If $G$ is integrable, the space $\left\{\phi_{-} \in \dot{L}_{p-}^{n}: G \phi_{-}=0\right\}$ is closed in $L_{p}^{n}$. One can consider the Riemann problem on the space

$$
\mathcal{X}_{p}=L_{p+}^{m}+\frac{\left\{\phi_{-} \in \dot{L}_{p-}^{n}: G \phi_{-} \in L_{p}^{m}\right\}}{\left\{\phi_{-} \in \dot{L}_{p-}^{n}: G \phi_{-}=0\right\}} .
$$

The kernel of the Riemann problem viewed on the space $\mathcal{X}_{p}$ can be finite dimensional even though $G$ is singular valued. Similarly, there may be a generically infinite 
dimensional co-image of the problem. The codimension of $\left\{\psi_{+} \in L_{q+}^{m}: G^{T} \psi_{+}=0\right\}$ in the annihilator of the image of the problem is independent of the left kernel of $G$.

By the defect numbers of a Riemann problem with coefficient $G$ we will understand the dimension $\alpha_{R}$ of the kernel of the problem in the space $\mathcal{X}_{p}$ and the co-dimension $\beta_{R}$ of the closure of the image of the problem in the space

$$
\mathcal{Y}_{p}^{m}=\left\{g \in L_{p}^{m}: \quad\left\langle\psi_{+}, g\right\rangle=0 \text { for all } \psi_{+} \in L_{q+}^{m} \text { such that } G^{T} \psi_{+}=0\right\} .
$$

The space $\mathcal{Y}_{p}^{m} \subset L_{p}^{m}$ is the intersection of closed subspaces, and so it is complete. If $\alpha_{R}$ or $\beta_{R}$ is finite, the difference $\alpha_{R}-\beta_{R}$ will be called the index of the problem. Note that $\alpha_{R}$ can also be defined as the dimension of the space of "+" components of elements in the kernel of the problem (viewed either on the space $\mathcal{X}_{p}$ or $L_{p+}^{m} \dot{+} \dot{L}_{p-}^{n}$ ).

The defect numbers of the dual problem are the dimension $\alpha_{D}$ of kernel of the dual problem in the space

$$
\widehat{\mathcal{X}}_{q}=\dot{L}_{q-}^{n}+\frac{\left\{\psi_{+} \in L_{q+}^{m}: G^{T} \psi_{+} \in L_{q}^{n}\right\}}{\left\{\psi_{+} \in L_{q+}^{m}: G^{T} \psi_{+}=0\right\}},
$$

and the co-dimension $\beta_{D}$ of the closure of the image of the dual problem in

$$
\widehat{\mathcal{Y}}_{q}^{n}=\left\{h \in L_{q}^{n}: \quad\left\langle\phi_{-}, h\right\rangle=0 \text { for all } \phi_{-} \in \dot{L}_{p-}^{n} \text { such that } G \phi_{-}=0\right\} .
$$

The number $\alpha_{D}$ can be equivalently defined as the dimension of the space of " - " components of elements in the kernel of the dual problem. If $\alpha_{D}$ or $\beta_{D}$ is finite, the difference $\alpha_{D}-\beta_{D}$ is called the index of the dual problem.

Proposition 4.1. If $G$ admits a spectral factorization in $L_{p}$,

$$
\alpha_{R}=\beta_{D}=\sum\left\{\kappa_{i}: \kappa_{i}>0\right\} \quad \text { and } \quad \alpha_{D}=\beta_{R}=\sum\left\{-\kappa_{j}: \kappa_{j}<0\right\} .
$$

In particular, all defect numbers are finite and the index of the problem (resp. the dual problem) is equal to the total index (resp. the opposite of the total index) of the factorization.

Proof. Let $G=G_{+} \Lambda G_{-}$with $\Lambda$ as in (2.3) be a spectral factorization in $L_{p}$. Then

$$
\alpha_{R}=\operatorname{dim}\left(\left(\Lambda G_{-}\left(\dot{L}_{p-}^{n}\right)\right) \cap L_{1+}^{m}\right) \quad \text { and } \quad \alpha_{D}=\operatorname{dim}\left(\left(\Lambda^{T} G_{+}^{T}\left(L_{q+}^{m}\right)\right) \cap \dot{L}_{1-}^{n}\right),
$$

and the statement about $\alpha_{R}$ and $\alpha_{D}$ follows.

The space $\mathcal{Y}_{p}^{m}$ coincides with (3.10). Indeed, $\mathcal{Y}_{p}^{m}$ clearly contains (3.10). Since the closure of (3.11) annihilates $\mathcal{Y}_{p}^{m}$, the reverse inclusion also holds. Similarly, $\widehat{\mathcal{Y}}_{q}^{n}$ coincides with the space of $h \in L_{q}^{n}$ which satisfy (3.9), and so the statement regarding $\beta_{R}$ and $\beta_{D}$ follows from Theorem 3.3.

Theorem 3.3 yields the following sufficient condition for normal solvability of the Riemann problem with factorable coefficient.

Proposition 4.2. Suppose $G=G_{+} \Lambda G_{-}$is a spectral factorization in $L_{p}$ and the operators

$$
K_{+}=G_{+} \mathcal{P} G_{+}^{-1}: \quad \mathcal{Y}_{p}^{m} \rightarrow L_{p}^{m} \quad \text { and } \quad K_{-}=G_{-}^{-1} \Lambda^{\dagger} \mathcal{Q} G_{+}^{-1}: \quad \mathcal{Y}_{p}^{m} \rightarrow L_{p}^{n}
$$

are bounded. Then the image of the Riemann problem with coefficient $G$ is closed. 
Proof. Suppose that for all $g \in \mathcal{Y}_{p}^{m}$

$$
G_{+}^{-1} g \in L_{1}^{m} \dot{+} \dot{L}_{1-}^{m}, \quad G_{+} \mathcal{P} G_{+}^{-1} g \in L_{p+}^{m} \quad \text { and } \quad G_{-}^{-1} \Lambda^{\dagger} \mathcal{Q} G_{+}^{-1} g \in L_{p}^{n},
$$

and let $g$ belong to the closure of the image of the problem. By Theorem 3.3, (3.1) holds. By Theorem 3.3 and Lemma 3.1 in [6], (3.2') holds. Thus, $g$ belongs to the image of the problem and it follows that the image of the problem is closed.

In particular, the image of the problem is closed when the operators $K_{+}$and $K_{-}$ are bounded on the whole space $L_{p}^{m}$.

We note that even though the function $G$ is singular valued, the Riemann problem from the space $\mathcal{X}_{p}$ to the Banach space $\mathcal{Y}_{p}^{m}$ can be Fredholm. Extending the definition of $\Phi$-factorization introduced in the nonsingular case in [9], one may call a spectral factorization $G=G_{+} \Lambda G_{-}$for which operators (4.7) are bounded Fredholm, and a function which admits a Fredholm factorization can be called Fredholm factorable. Unlike in the nonsingular case, spectral factorization in $L_{p}$ of a Fredholm factorable function need not be Fredholm (see the example after Proposition 3.1). By Proposition 4.2, the Riemann problem with a Fredholm factorable coefficient is normally solvable.

If $G$ takes injective values, or if $G_{-}^{ \pm 1}$ are bounded, a stronger statement is true.

Theorem 4.3. Suppose $G=G_{+} \Lambda G_{-}$is a spectral factorization in $L_{p}$ and at least one of the following two conditions holds:

(i) $G$ takes injective values a.e. on $\Gamma$,

(ii) the matrix functions $G_{-}$and $G_{-}^{-1}$ are essentially bounded.

Then the image of the Riemann problem with coefficient $G$ is closed if and only if the operators

$$
K_{+}=G_{+} \mathcal{P} G_{+}^{-1}: \quad \mathcal{Y}_{p}^{m} \rightarrow L_{p}^{m} \quad \text { and } \quad K_{-}=G_{-}^{-1} \Lambda^{\dagger} \mathcal{Q} G_{+}^{-1}: \quad \mathcal{Y}_{p}^{m} \rightarrow L_{p}^{n}
$$

are bounded.

Proof. Sufficiency follows from Proposition 4.2. Suppose the image of the problem is closed, so that it coincides with the space of functions $g \in L_{p}^{m}$ which satisfy (3.6) and (3.7). By Proposition 3.1 (and the assumptions regarding $G_{-}^{ \pm 1}$ or the rank of $G$ ), the operators $K_{+}$and $K_{-}$are defined on the image of the problem. Since one can find a basis for a complement of the image of the problem in $\mathcal{Y}_{p}^{m}$ consisting of rational vector functions (cf. formula (3.6)), and $K_{ \pm}$are defined for all rational vector functions analytic on $\Gamma, K_{ \pm}$are defined on the whole $\mathcal{Y}_{p}^{m}$. Since the operators $K_{ \pm}$are closed, they are necessarily continuous.

In particular, if $G$ takes nonsingular values a.e. on $\Gamma, \mathcal{Y}_{p}^{m}=L_{p}^{m}$ and Theorem 4.3 reduces to a known result (Theorem 4.1 in [9]). Note that condition (i) in Theorem 4.3 implies $m \geq n$. If $m>n$, the system of integral equations given in the introduction is "overdetermined."

Suppose $G_{+} \Lambda G_{-}$is a spectral factorization in $L_{p}$ of a bounded matrix function $G$ and the operator $K_{-}$in (4.9) is continuous. Then the operator $G K_{-}=$ $G_{+} \Lambda \Lambda^{\dagger} \mathcal{Q} G_{+}^{-1}=G_{+} \mathcal{Q} G_{+}^{-1}$ is bounded on $\mathcal{Y}_{p}^{m}$, and it follows that $K_{+}$is continuous. That is, if a bounded matrix function $G$ admits a spectral factorization in $L_{p}$, and at least one of the conditions (i) and (ii) in Theorem 4.3 holds, then the image of the Riemann problem with coefficient $G$ is closed if and only if the operator $K_{-}$in (4.9) is continuous. 
Theorem 4.3 has the following immediate corollary.

Corollary 4.4. Suppose $G \neq 0$ admits a spectral factorization $G_{+} \Lambda G_{-}$in $L_{p}$ for any $p>1$ with $G_{+}, G_{-}$, and $G_{-}^{-1}$ essentially bounded, and the Riemann problem with coefficient $G$ is normally solvable. Then the contour $\Gamma$ is regular (and hence Smirnov).

Proof. If $G_{+}$is bounded,

$$
G_{+}\left[\begin{array}{c}
L_{p} \\
L_{p+}^{(m-1) \times 1}
\end{array}\right] \subset \mathcal{Y}_{p}^{m} .
$$

Since $K_{+}$in (4.9) is bounded, $\mathcal{P}$ is a bounded projection.

Since each rational matrix function without poles or zeros on $\Gamma$ admits a spectral factorization in $L_{p}$ with all factors (and their multiplicative inverses) essentially bounded, Theorem 4.3 has also the following corollary.

Corollary 4.5. Let $G \neq 0$ be a rational matrix function without poles or zeros on $\Gamma$. Then the image of the Riemann problem with coefficient $G$ is closed if and only if $\Gamma$ is regular.

Proposition 4.2 and Theorem 4.3 have their analogue in the dual space.

Theorem 4.6. Suppose $G_{+} \Lambda G_{-}$is a spectral factorization in $L_{p}$ of an $m \times n$ matrix function $G$. If the operators

$$
\widehat{K}_{-}=G_{-}^{T} \mathcal{Q} G_{-}^{-T}: \widehat{\mathcal{Y}}_{q}^{n} \rightarrow L_{q}^{n} \quad \text { and } \quad \widehat{K}_{+}=G_{+}^{-T} \Lambda^{\dagger T} \mathcal{P} G_{-}^{-T}: \widehat{\mathcal{Y}}_{q}^{n} \rightarrow L_{q}^{m}
$$

are bounded, then the image of the dual problem is closed. Conversely, if the image of the dual problem is closed, and

(i) the matrix functions $G_{+}$and $G_{+}^{-1}$ are essentially bounded, or

(ii) $G$ takes surjective values a.e. on $\Gamma$,

then the operators $\widehat{K}_{-}$and $\widehat{K}_{+}$are bounded.

Note that if $G$ is bounded then, by Proposition 3.2 , the continuity of $\widehat{K}_{+}$in (4.11) implies the continuity of $\widehat{K}_{-}$. Thus, if condition (i) or (ii) in Theorem 4.6 holds, the dual problem is normally solvable if and only if $\widehat{K}_{+}$is bounded.

It follows from Theorem 4.6 that if $G \neq 0$ admits a spectral factorization in $L_{p}$ with the factors $G_{-}, G_{+}$, and $G_{+}^{-1}$ essentially bounded, and the image of the dual problem is closed, then the contour is regular. Also, if $G$ is a nonzero rational matrix function without poles or zeros on $\Gamma$, then the image of the dual problem is closed if and only if $\Gamma$ is regular.

Theorems 4.3 and 4.6 have the following corollary.

Theorem 4.7. Suppose $G$ is a bounded matrix function which admits a spectral factorization in $L_{p}$, at least one of conditions (i)-(ii) in Theorem 4.3 holds, and at least one of conditions (i)-(ii) in Theorem 4.6 holds. Then the Riemann problem with coefficient $G$ is normally solvable if and only if the dual problem is normally solvable.

Proof. Since

$$
K_{-} G_{+}\left[\begin{array}{c}
0 \\
\dot{L}_{\infty-}^{m-k}
\end{array}\right]=(0) \quad \text { and } \quad \widehat{K}_{+} G_{-}^{T}\left[\begin{array}{c}
0 \\
L_{\infty+k}^{n-k}
\end{array}\right]=(0),
$$


the domains of $K_{-}$and $\widehat{K}_{+}$can be extended to linear manifolds

$$
\mathcal{Y}_{p, E}^{m}:=\mathcal{Y}_{p}^{m}+G_{+}\left[\begin{array}{c}
0 \\
\dot{L}_{\infty-}^{m-k}
\end{array}\right] \quad \text { and } \quad \widehat{\mathcal{Y}}_{q, E}^{n}:=\widehat{\mathcal{Y}}_{q}^{n}+G_{-}^{T}\left[\begin{array}{c}
0 \\
L_{\infty+k}^{n-k}
\end{array}\right] \text {. }
$$

Let $\tilde{K}_{-}=G_{-}^{-1} \mathcal{Q} \Lambda^{\dagger} G_{+}^{-1}: \mathcal{Y}_{p, E}^{m} \rightarrow L_{p}^{m}$. Since $\mathcal{P}$ is the adjoint of $\mathcal{Q},\left\langle\tilde{K}_{-} f, g\right\rangle=$ $\left\langle f, \widehat{K}_{+} g\right\rangle$ for all $f \in \mathcal{Y}_{p, E}^{m}$ and $g \in \widehat{\mathcal{Y}}_{q, E}^{n}$. It follows that $\tilde{K}_{-}$and $\widehat{K}_{+}$are adjoints. Since $\mathcal{Y}_{p, E}^{m}$ and $\widehat{\mathcal{Y}}_{q, E}^{n}$ are dense in $L_{p}^{m}$ and $L_{q}^{n}$, respectively, and $\tilde{K}_{-}$and $\widehat{K}_{+}$satisfy equations (4.12), $\tilde{K}_{-}$is bounded on $\mathcal{Y}_{p}^{m}$ if and only if $\widehat{K}_{+}$is bounded on $\widehat{\mathcal{Y}}_{q}^{n}$. Since $G_{-}^{-1} \mathcal{P} \Lambda^{\dagger} \mathcal{Q} G_{+}^{-1}$ and $G_{-}^{-1} \mathcal{Q} \Lambda^{\dagger} \mathcal{P} G_{+}^{-1}$ are finite rank bounded operators from $L_{p}^{m}$ to $L_{p}^{n}$, and $K_{-}=\tilde{K}_{-}+G_{-}^{-1} \mathcal{P} \Lambda^{\dagger} \mathcal{Q} G_{+}^{-1}-G_{-}^{-1} \mathcal{Q} \Lambda^{\dagger} \mathcal{P} G_{+}^{-1}$, the boundedness of the operator $\tilde{K}_{-}$is equivalent to the boundedness of $K_{-}$.

The special case of Theorem 4.7 where $G$ takes nonsingular values a.e. on $\Gamma$ has been known previously.

\section{REFERENCES}

1. J. A. Ball and J. W. Helton, Beurling-Lax Representations Using Classical Lie Groups with Many Applications II: $G L(n, \mathbf{C})$ and Wiener-Hopf Factorization, Int. Equ. and Op. Th. 7 (1984), 291-309. MR 86e:47020

2. K. Clancey and I. Gohberg, Factorization of Matrix Functions and Singular Integral Operators, OT 3, Birkhäuser Verlag, Basel/Boston/Stuttgart, 1981. MR 84a:47016

3. K. Clancey and M. Rakowski, Factorization of Rectangular Matrix Functions Relative to a Contour, manuscript, 1990.

4. G. David, Opérateurs intégraux singuliers sur certains courbes du plan complexe, Ann. Scient. Éc. Norm. Sup. 17 (1984), 157-189. MR 85k:42026

5. P. Duren, Theory of $H^{p}$ spaces, Academic Press, New York, 1970. MR 42:3552

6. G. S. Litvinchuk and I. M. Spitkovsky, Factorization of Measurable Matrix Functions, OT 25, Birkhäuser Verlag, Basel/Boston, 1987. MR 90g:47030

7. M. Rakowski, Spectral Factorization of Rectangular Rational Matrix Functions with Application to Discrete Wiener-Hopf Equations, J. Functional Analysis 10 (1992), 410-433. MR 93j: 47031

8. M. Rakowski and I. Spitkovsky, Spectral Factorization of Measurable Rectangular Matrix Functions and the Vector Valued Riemann Problem, Revista Matemática Iberoamericana, to appear.

9. I. Spitkovsky, Factorization of Measurable Matrix-Value Functions and its Relation to the Theory of Singular Integral Equations and the Vector Riemann Boundary-Value Problem, I, English translation: Differential Equations 17 (1981), 477-485.

Department of Mathematics, The Ohio State University, Columbus, Ohio 43210

E-mail address: rakowski@math.ohio-state.edu

Department of Mathematics, The College of William and Mary, Williamsburg, VirGINIA 23187-8795

E-mail address: ilya@cs.wm.edu 